\title{
A Combinatorial Property of Points and Ellipsoids
}

\author{
I. Bárány* and D. G. Larman \\ Department of Mathematics, University College London, \\ Gower Street, London WC1E 6BT, England
}

\begin{abstract}
For each $d \geq 1$ there is a constant $c_{d}>0$ such that any finite set $X \subset R^{d}$ contains a subset $Y \subset X,|Y| \leq\left\lfloor\frac{1}{4} d(d+3)\right\rfloor+1$ having the following property: if $E \supset Y$ is an ellipsoid, then $|E \cap X| \geq c_{d}|X|$.
\end{abstract}

\section{Introduction and Results}

A nice and recent result of Neumann-Lara and Urrutia [4] states that any finite point-set $X$ in the plane contains two points such that every circle containing these two contains $1.5 \%$ of the points of $X$. This result has been extended to the $d$-dimensional Euclidean space $R^{d}$ in [1] in the following form: any finite set $X \subset R^{d}$ contains a subset $Y \subset X,|Y| \leq\left\lfloor\frac{1}{2}(d+3)\right\rfloor$ such that if $B \subset R^{d}$ is a ball with $Y \subset B$, then $|B \cap X| \geq c_{d}|X|$, where $c_{d}$ is a constant depending on $d$ only. It is also shown in [1] that the bound $\left\lfloor\frac{1}{2}(d+3)\right\rfloor$ on the size of $Y$ cannot be decreased.

The aim of this paper is to extend the above results to ellipsoids and, more generally, to quadrics in $R^{d}$. We also hope that our proofs will give insight as to why these properties of points and balls or points and ellipsoids hold.

Before stating our theorems we fix the necessary notation and terminology. We assume that a coordinate system is given in $R^{d}$ so a point $x \in R^{d}$ can be written as $x=\left(x_{1}, x_{2}, \ldots, x_{d}\right)$. Let $P$ be a $d \times d$ symmetric matrix, $p \in R^{d}$ and $p_{0} \in R$. Then the quadric $Q=Q\left(P, p, p_{0}\right)$ is the set of points $x \in R^{d}$ satisfying

$$
x^{T} P x+p^{T} x+p_{0} \leq 0 .
$$

* On leave from the Mathematical Institute of the Hungarian Academy of Sciences, 1364 Budapest, P.O. Box 127, Hungary. Supported by a research fellowship from the Science and Engineering Research Council, U.K., and by Hungarian National Foundation for Scientific Research Grant No. 1812. 
An ellipsoid is a quadric with $P$ positive definite.

A key step in our proofs is another interpretation of quadrics. To define this consider first the map $v: R^{d} \rightarrow R^{m}$ with $m=(d+1)(d+2) / 2$ that maps $x=$ $\left(x_{1}, \ldots, x_{d}\right)$ to

$$
v(x)=\left(1, x_{1}, \ldots, x_{d}, x_{1}^{2}, x_{1} x_{2}, \ldots, x_{1} x_{d}, x_{2}^{2}, x_{2} x_{3}, \ldots, x_{d}^{2}\right) .
$$

Then a quadric $Q$ in $R^{d}$ can be written as

$$
Q=\left\{x \in R^{d}: q^{T} v(x) \leq C\right\}
$$

where $q=\left(q_{0}, q_{1}, \ldots, q_{d}, q_{11}, q_{12}, \ldots, q_{1 d}, q_{22}, q_{23}, \ldots, q_{d d}\right) \in R^{m}$ is suitably chosen. Clearly, $q$ determines $P, p$, and $p_{0}$ and vice versa (up to a positive multiplier).

Theorem 1. For $d=1,2, \ldots$ there is a constant $c_{d}>0$ such that any finite set $X \subset R^{d}$ contains a subset $Y \subset X,|Y| \leq\left\lfloor\frac{1}{4} d(d+3)\right\rfloor+1$ having the property that if a quadric $Q$ contains $Y$, then $|Q \cap X| \geq c_{d}|X|$.

Theorem 2. There is an infinite set $X \subset R^{d}$ such that for any $Y \subset X,|Y|=$ $\left\lfloor\frac{1}{4} d(d+3)\right\rfloor$ there is a quadric $Q$ with $Q \cap X=Y$.

This shows that in Theorem 1 the bound $\left\lfloor\frac{1}{4} d(d+3)\right\rfloor+1$ on the size of $Y$ is best possible. Let us have a more general look at the problem. Let $\mathscr{F}$ be a family of sets in $d$-space and define $t=t(\mathscr{F})$ as the smallest integer for which there exists a positive number $c$ such that every finite set $X \subset R^{d}$ contains a subset $Y \subset X,|Y| \leq t$, with the property that $F \in \mathscr{F}$ and $Y \subset F$ imply $|F \cap X| \leq c|X|$. (If no such integer exists, then define $t=\infty$.) Theorems 1 and 2 show that $t(F)=$ $\left\lfloor\frac{1}{4} d(d+3)\right\rfloor+1$ when $\mathscr{F}$ is the family of quadrics in $R^{d}$. For the class of ellipsoids we have a weaker result: define $k(2)=2, k(3)=4$, and $k(d)=\left\lfloor\frac{1}{4} d(d+1)+\frac{1}{2}\right\rfloor$ for $d \geq 4$.

Theorem 3. There is an infinite set $X \supset R^{d}$ such that for any $Y \subset X,|Y|=k(d)$ there is an ellipsoid $E$ with $E \cap X=Y$.

Denote the family of all ellipsoids in $R^{d}$ by $\mathscr{E}^{d}$. Then Theorems 1 and 3 show that $t\left(\mathscr{E}^{2}\right)=3, t\left(\mathscr{E}^{3}\right)=5$, and $\left\lfloor\frac{1}{4} d(d+1)+\frac{1}{2}\right\rfloor<t\left(\mathscr{E}^{d}\right) \leq\left\lfloor\frac{1}{4} d(d+3)\right\rfloor+1$. The two authors disagree about which bound is nearer to $t\left(\mathscr{E}^{d}\right)$.

\section{Proof of Theorem 1}

We start with a lemma similar to the one in [4] and [1].

Lemma 4. If $Z \subset R^{d}$ and $|Z|=m+1$, then there is $Y \subset Z,|Y| \leq \frac{1}{2}(m+1)$ such that, for any quadric $Q$ with $Y \subset Q$, the set $Q \cap(Z \backslash Y)$ is nonempty. 
Proof. As $Z$ contains $m+1$ points, the vectors $v(x) \in R^{m}(x \in Z)$ are linearly dependent. This can be written (by separating the positive and negative coefficients) as

$$
\begin{gathered}
\sum_{x \in Y} \alpha(x) v(x)=\sum_{x \in \mathcal{Z} \backslash Y} \beta(x) v(x), \\
\alpha(x) \geq 0, \quad \beta(x) \geq 0, \quad \alpha \neq 0 \text { or } \beta \neq 0 .
\end{gathered}
$$

We may and do name $Y$ so as to satisfy $|Y| \leq|Z \backslash Y|$. We now claim that $Y$ has the property stated in the lemma. Clearly, $|Y| \leq \frac{1}{2}(m+1)$. Assume $Q$ is a quadric containing $Y$, i.e.,

$$
q^{T} v(x) \leq 0 \quad \text { for } \quad x \in Y
$$

with $q$ coming from (2). Multiply the last inequality by $\alpha(x)$ and sum for $x \in Y$, then we get, using (3),

$$
\begin{aligned}
0 & \geq \sum_{x \in Y} \alpha(x) q^{T} v(x)=q^{T} \sum_{x \in Y} \alpha(x) v(x)=q^{T} \sum_{x \in Z \backslash Y} \beta(x) v(x) \\
& =\sum_{x \in Z \backslash Y} \beta(x) q^{T} v(x) .
\end{aligned}
$$

Assume now that $Q \cap(Z \backslash Y)$ is empty, i.e., $q^{T} v(x) \leq 0$ for all $x \in Z \backslash Y$. Then $\beta(x)=0$ must hold for all $x \in Z \backslash Y$. The first component of $v(x)$ is always 1 and comparing the first components in (3) we get that $\alpha(x)=0$ for all $x \in Y$, a contradiction.

Now we turn to the proof of Theorem 1. We use the same counting argument as in [4] and [1]. Call a pair $(Z, Y)$ good if it satisfies Lemma 4, that is, $Y \subset Z \subset X$, $|Y| \leq \frac{1}{2}(m+1),|Z|=m+1$ and for any quadric $Q$ with $Y \subset Q$, the set $Q \cap(Z \backslash Y)$ is nonempty. Write $|X|=n$ and assume that $n \geq 2 \mathrm{~m}$. We count the number of good pairs in two ways:

$$
\text { number of good pairs }=\sum_{(Z, Y)_{\text {good }}} 1=\sum_{\substack{Z \in X \\
|Z|=m+1}} \sum_{\substack{(Z, Y) \text { good } \\
Y \in Z}} 1 \geq\left(\begin{array}{c}
n \\
m+1
\end{array}\right)
$$

according to Lemma 4.

Now for $Y \subset X,|Y| \leq \frac{1}{2}(m+1)$ write $t(Y)=\min \{|Q \cap X|: Q$ is a quadric with $Y \subset Q\}$ and $T(Y)=Q \cap X$ with the quadric $Q$ for which the minimum is attained. We have to prove that $t(Y) \geq c_{d}|X|$ for some $Y$ so we define, further, $t=$ $\max \left\{t(Y): Y \subset X,|Y| \leq \frac{1}{2}(m+1)\right\}$ :

$$
\text { number of good pairs }=\sum_{\substack{(Z, Y) \\ \text { good }}} 1=\sum_{i=0}^{(m+1) / 2} \sum_{\substack{Y \subset X X \\|Y|=i}} \sum_{(Z,|Z|=m+1} 1 .
$$


In the last sum $Z$ must contain a point from $T(Y) \backslash Y$ for otherwise $Q \cap(Z \backslash Y)$ would be empty for the quadric $Q$ with $T(Y)=Q \cap X$. So we can extend $Y$ to $Z$ in at most

$$
(t(Y)-|Y|)\left(\begin{array}{c}
n-|Y|-1 \\
m-|Y|
\end{array}\right) \leq t\left(\begin{array}{c}
n-i-1 \\
m-i
\end{array}\right)
$$

ways. Then

$$
\text { number of good pairs } \leq \sum_{i=0}^{(m+1) / 2}\left(\begin{array}{c}
n \\
i
\end{array}\right) t\left(\begin{array}{c}
n-i-1 \\
m-i
\end{array}\right) \leq \frac{t}{n}\left(\begin{array}{c}
n \\
m+1
\end{array}\right) m 2^{m+1}
$$

Comparing (4) and (5) we get $t \geq m^{-1} 2^{-(m+1)} n$.

We can see that this proof works in a more general setting. Instead of the quadrics, we take a family $\mathscr{F}$ of sets in $R^{d}$ generated by a finite set of functions $f_{1}, \ldots, f_{m}: R^{d} \rightarrow R$ with $f_{1}(x) \equiv 1$ ( $m$ need not be equal to $\frac{1}{2}(d+2)(d+1)$ here) in the following sense: any set $F \in \mathscr{F}$ can be written as

$$
F=\left\{x \in R^{d}: \sum_{i=1}^{m} \alpha_{i} f_{i}(x) \leq 0\right\}
$$

for suitable reals $\alpha_{1}, \ldots, \alpha_{m}$. In this setting the following theorem holds. Any finite set $X \subset R^{d}$ contains a subset $Y \subset X,|Y| \leq \frac{1}{2}(m+1)$ such that $F \in \mathscr{F}$ and $Y \subset F$ imply $|X \cap C| \geq c(m)|X|$ where $c(m)=m^{-1} 2^{-(m+1)}$ is a constant. In particular, we can get the results of [4] and [1] because Euclidean balls in $R^{d}$ can be generated by the functions $f_{1}(x)=1, f_{2}(x)=x_{1}, f_{3}(x)=x_{2}, \ldots, f_{d+1}(x)=x_{d}$, $f_{d+2}(x)=x_{1}^{2}+\cdots+x_{d}^{2}$.

We mention here that for $d=2$ Lemma 4 says that from seven points in the plane we can choose three such that any quadric containing these three contains a fourth. This can be strengthened for ellipsoids (we are not going to give the proof).

Lemma 4'. From six points in the plane we can always choose three so that any ellipsoid containing these three contains a fourth.

\section{The Example Proving Theorem 2}

We start with a lemma that follows from the well-known "Descartes" Rule of Signs." Here we give a simple proof.

Lemma 5 (see [3]). Let $S \subset\{0,1, \ldots\}$ be a finite set and assume that the polynomial $g(t)=\sum_{i \in S} g_{i} t^{i}$ is not identically zero. Then $g$ has at most $(|S|-1)$ positive roots, counted with multiplicities. 
Proof. We use an induction argument from [3]. When $|S|=1$, the statement is trivial. Assume it has been proved for $|S|<s \quad(s \geq 2)$ and we are going to prove it for $|S|=s$. Assume, on the contrary, that $g$ has $s$ positive roots. Set $j=\min \{i: i \in S\}$. Then the polynomial

$$
h(t)=t^{-j} g(t)=\sum_{i \in S} g_{i} t^{i-j}
$$

has $s$ positive roots, too. Now if $t_{1}<t_{2}$ are two such roots, then, by Rolle's theorem, there is a root of $h^{\prime}$ between $t_{1}$ and $t_{2}$. If $t_{0}$ is a root of $h$ with multiplicity $\mu(\mu>1)$, then $t_{0}$ is a root of $h^{\prime}$ with multiplicity $\mu-1$. So the polynomial

$$
h^{\prime}(t)=\sum_{i \in S \backslash\{j\}}(i-j) g_{i} t^{i-j-1}
$$

has $s-1$ roots, contradicting the induction hypothesis.

For the construction we will need a set $A \subset\{0,1,2, \ldots\}$ with $0 \in A,|A|=d+1$ such that for $a_{1}, a_{2}, a_{3}, a_{4} \in A$

$$
a_{1}+a_{2}=a_{3}+a_{4} \quad \text { if and only if }\left\{a_{1}, a_{2}\right\}=\left\{a_{3}, a_{4}\right\}
$$

Such a set exists, take $A=\left\{0,1,3,9, \ldots, 3^{d-1}\right\}$, for instance. There are other examples as well, see [2] for one with $A \subset\left\{0,1, \ldots, d^{3}\right\}$. The property of $A$ we will need is that the set $A+A=\{a+b: a \in A, b \in A\}$ is of size $m=\frac{1}{2}(d+1)(d+2)$.

Now let $A=\left\{a_{0}=0<a_{1}<\cdots<a_{d}\right\}$ be such a set and define (see [3]) the generalized moment curve $x(t) \in R^{d}$ for $t \in R$ as

$$
x(t)=\left(t^{a_{1}}, t^{a_{2}}, \ldots, t^{a_{d}}\right)
$$

Let $k=\left\lfloor\frac{1}{4} d(d+3)\right\rfloor$. The set $X=\{x(t): t>0\}$ will be the example proving Theorem 2. This is shown by

Claim 6. For $0<t_{1}<t_{2}<\cdots<t_{k}$ there is a quadric $Q$ such that

$$
Q \cap X=\left\{x\left(t_{1}\right), \ldots, x\left(t_{k}\right)\right\}
$$

Proof. Define

$$
B= \begin{cases}A+A & \text { if }\left(\begin{array}{c}
d+2 \\
2
\end{array}\right) \text { is odd, } \\
(A+A) \backslash\{0\} & \text { otherwise. }\end{cases}
$$

It is easy to see that

$$
2 k+1=|B|
$$


Now we construct a polynomial $f(t)=\sum_{i \in B} f_{i} t^{i}$ with the condition that $t_{j}$ is a root of multiplicity 2 of $f$ :

$$
\begin{aligned}
\sum_{i \in B} f_{i} t_{j}^{i}=0 & \text { for } j=1, \ldots, k \\
\sum_{i \in B} i f_{i} t_{j}^{i-1}=0 & \text { for } j=1, \ldots, k
\end{aligned}
$$

This is a system of linear homogeneous equations that has a solution because, by $(7)$, the number of equations, $2 k$, is just one less than the number of unknowns, $|B|$. So let $f_{i}(i \in B)$ be a nontrivial solution to $(8)$.

Now $f(t)$ has at least $2 k$ positive roots and, by Lemma 5 , it has at most $|B|-1$ positive roots. So it has exactly $k$ positive roots $t_{1}, \ldots, t_{k}$ each with multiplicity

2. Then multiplying with -1 if necessary we assume that $f(t) \geq 0$ for $t \geq 0$.

We look for the quadric $Q$ in the form (2)

$$
Q=\left\{x \in R^{d}: q^{T} v(x) \leq 0\right\}
$$

where $q \in R^{m}$. We determine $q$ by requiring that

$$
q^{T} v(x(t)) \equiv f(t)
$$

This is possible because the components of $v(x(t))$ are of the form $t^{a}$ with $a \in A+A \subset B$. The quadric $Q$ defined in this way has the required properties: the curve $x(t)$ has a twofold touching with $Q$ at $t=t_{1}, \ldots, t_{k}$ from outside and is disjoint from $Q$ for $t>0, t \neq t_{i}(i=1, \ldots, k)$.

Combining the proofs of Theorems 1 and 2 we can prove the following theorem. Let $\mathscr{F}_{r}^{d}$ denote the family of all sets $F \subset R^{d}$ that can be written as

$$
F=\left\{x \in R^{d}: f(x) \leq 0\right\}
$$

where $f$ is an at most $r$-degree polynomial of the variables $x_{1}, \ldots, x_{d}$.

Theorem. $\quad t\left(\mathscr{F}_{r}^{d}\right)=\left[\frac{1}{2}\left(\begin{array}{c}d+r \\ r\end{array}\right)+\frac{1}{2}\right]$

\section{The Example Proving Theorem 3}

This is similar to the previous example. We start with the case $d>3$ and set $k=k(d)=\left\lfloor\frac{1}{4} d(d+1)+\frac{1}{2}\right\rfloor$. Define

$$
x(t)=\left(t, t^{2}, t^{4}, t^{8}, \ldots, t^{2^{d-1}}\right)
$$


so $A=\left\{0,2^{0}, 2^{1}, \ldots, 2^{d-1}\right\}$ and this time condition (6) does not hold. Still, $A+A=$ $\left\{0,2^{0}, 2^{1}, \ldots, 2^{d}, \ldots, 2^{i}+2^{j}, \ldots\right\}$ and only the powers $2^{i}(i=1,2, \ldots, d-1)$ have double representation in $A+A: 2^{i}=0+2^{i}=2^{i-1}+2^{i-1}$. Clearly, $|A+A|=$ $\frac{1}{2} d(d+1)+2$. Set, again, $B=A+A$ if $\frac{1}{2} d(d+1)$ is odd and $B=(A+A) \backslash\{0\}$ otherwise. Then

$$
2 k+1=|B| \text {. }
$$

Claim 7. For $0<t_{1}<t_{2}<\cdots<t_{k}$ there is an ellipsoid $E$ with

$$
E \cap\{x(t): t>0\}=\left\{x\left(t_{1}\right), \ldots, x\left(t_{k}\right)\right\}
$$

Proof. Construct a polynomial $f(t)=\sum_{i \in B} f_{i} t^{i}$ having double zeros at $t=$ $t_{1}, \ldots, t_{k}$. This can be done in the same way as before. Moreover, again by Lemma $5, f(t)$ has no other positive root and we may assume that $f(t) \geq 0$ for $t \geq 0$. Then the leading coefficient of $f$ is positive. By Lemma 5, again, no $f_{i}(i \in B)$ equals zero, so the leading coefficient of $f$ is

$$
f_{2^{d}}>0
$$

This time we look for the ellipsoid $E$ in the form

$$
x^{T} P x+p^{T} x+p_{0} \leq 0
$$

with the requirement that

$$
x^{T}(t) P x(t)+p^{T} x(t)+p_{0} \equiv f(t)
$$

From here we get all the off-diagonal entries of $\boldsymbol{P}$ and $p_{d d}$ and $p_{1}, p_{3}, p_{5}, \ldots$, and $p_{0}$ explicitly, in particular,

$$
p_{d d}=f_{2^{d}}>0
$$

Further, we get $d-1$ equations for the remainder of the unknowns:

$$
p_{i i}+p_{i+1}=f_{2^{\prime}}, \quad i=1,2, \ldots, d-1
$$

We use the freedom for the choice of $p_{i i}$ to ensure that $P$ is positive definite. All we need is the positivity of the determinants

$$
p_{d d}, \quad\left|\begin{array}{cc}
p_{d-1, d-1} & p_{d-1, d} \\
p_{d, d-1} & p_{d d}
\end{array}\right|,\left|\begin{array}{ccc}
p_{d-2, d-2} & p_{d-2, d-1} & p_{d-2, d} \\
p_{d-1, d-2} & p_{d-1, d-1} & p_{d-1, d} \\
p_{d, d-2} & p_{d, d-1} & p_{d d}
\end{array}\right|, \ldots
$$


These are the main minors of $P$ starting from the lower-right corner. We proved in (9) that $p_{d d}>0$. Then, by an easy inductive construction, we find $p_{d-1, d-1}$, $p_{d-2, d-2}, \ldots, p_{11}$ so that these determinants are all positive. The values $p_{i+1}$ can be recovered from $(10)$.

Thus the quadric $x^{T} P x+p^{T} x+p_{0} \leq 0$ defines an ellipsoid $E$ and we finish the proof of the claim the same way as before.

We are left with the cases $d=2,3$. The former is trivial so consider $d=3$. The set $X$ will be $\{x(t):|t-1|<\eta\}$ with $\eta>0$ small where

$$
x(t)=\left(t^{2}, t^{4}, t^{5}\right) \in R^{3} .
$$

Here $A=\{0,2,4,5\}$ and $B=A+A=\{0,2,4,5,6,7,8,9,10\}$. First we find a polynomial $f(t)=\sum_{i \in B} f_{i} t^{i}$ that has a zero of multiplicity 8 at $t=1$ :

$$
f(t)=(t-1)^{8}\left(g_{0}+g_{1} t+g_{2} t^{2}\right) .
$$

A suitable solution is $g_{0}=1, g_{1}=8, g_{2}=21$. This defines the coefficients $f_{i}(i \in B)$ explicitly. We find a quadric $Q=Q\left(P, p, p_{0}\right)$ through the condition

$$
x^{T}(t) P x(t)+p^{T} x(t)+p_{0} \equiv f(t) .
$$

This gives

$$
P=\left[\begin{array}{crr}
p_{11} & 525 & -480 \\
525 & 525 & -80 \\
-480 & -80 & 21
\end{array}\right], \quad p^{T}=\left[\begin{array}{c}
-15 \\
p_{2} \\
-627
\end{array}\right], \quad p_{0}=1
$$

with the condition $p_{11}+p_{2}=210$. We choose $p_{11}=18688$ (or anything larger), then $p_{2}=-18478$, and $P$ is positive definite because its main minors are all positive. Then the quadric $Q\left(P, p, p_{0}\right)$ is an ellipsoid $E$ that has an eightfold touching with the curve $x(t)$ at $t=1$ from outside.

Since the numbers and results are stable under a small perturbation, there exists $\eta>0$ such that if $t_{1}, t_{2}, t_{3}, t_{4}$ are chosen within $\eta$ of $t=1$, then there is an ellipsoid $E$ that has a twofold touching with $x(t)$ at $t=t_{i}, i=1,2,3,4$. Moreover, it follows that

$$
E \cap\{x(t):|t-1|<\eta\}=\left\{x\left(t_{i}\right): i=1,2,3,4\right\} .
$$

\section{References}

1. I. Bárány, J. H. Schmerl, S. J. Sidney, and J. Urrutia, A combinatorial result about points and balls in Euclidean space, Discrete Comput. Geom. 4 (1989), 259-262.

2. P. Erdös and P. Turán, On a problem of Sidon in additive number theory and some other problems, J. London Math. Soc. 16 (1941), 212-215; Addendum, ibid. 19 (1944), 208.

3. F. R. Gantmacher, The Theory of Matrices, New York, Chelsea, 1959.

4. V. Neumann-Lara and J. Urrutia, A combinatorial result on points and circles in the plane, Report TR-85-15, University of Ottawa, November, 1985. 